\title{
Prescription of Maintenance Interventions by the New Generation of Eurocodes for Climate-Change Resilient Structures
}

\author{
Maria Nogal $^{1}$ \\ ${ }^{1}$ Faculty of Civil Engineering \& Geosciences, Delft University of Technology, Netherlands \\ m.nogal@tudelft.nl
}

\begin{abstract}
The new generation of structural Eurocodes will include climate change adaption measures in order to enhance the climate resilience of infrastructures across Europe. This paper discusses the impact that climate change might have on reinforced concrete structures subjected to corrosion, and the associated challenges of the standardisation of adaptation measures. A resilience-based framework is proposed for the adaptation of structural codes to climate change-induced actions. Such an approach aims to provide the required adaptive capacity to the new structures in order to be able to respond to the uncertain future minimising the investment under unlikely scenarios. In that way, the strategy can be summarised as a climate change-adapted design plus an in-design maintenance plan. Future actions, such as mapping the future climate-related drivers of corrosion, the determination of the design values of these drivers, and the prescription of maintenance activities, should be further investigated. Although this paper focuses on the new structures that will be designed with the new codes, some of the insights can be extrapolated to the existing structures.
\end{abstract}

Keywords: Corrosion, Climate change, Resilience, Maintenance, Eurocodes.

\section{Introduction}

The pressure imposed by climate change has reached all sectors within the European Commission, which is now forced to take adaptation measures. In Union Innovation (2013), they acknowledged action gaps in a context where late responses could result in non-affordable economic, social, and environmental costs. Consequently, they proposed a road-map to include climate change adaptation considerations where technical standards were identified as an effective strategy to enhance the climate resilience of infrastructures across Europe.

In the construction sector, the first generation of EN Eurocodes was launched in 2007 with the aim to homogenise structural design within the Member States. The second generation of Eurocodes is planned by 2023, which will reflect the new market needs (e.g., new materials and construction techniques) and also the European commitment with the sustainable development goals. Therefore, the second generation of Eurocodes is planned to incorporate the adaptation of structural design to climate change.

Within the long list of impacts upon structures caused by climate change, corrosion has been identified as a priority theme, as it has been estimated that the increased maintenance and repair costs worldwide resulting from the acceleration of the corrosion process due to climate change might be of hundreds of billions of dollars annually (Bastidas-Arteaga \& Stewart (2015)). 
In this context, this paper discusses the adaptation strategy to be applied to the new generation of Eurocodes in relation to the corrosion of reinforced concrete (RC) structures. At present, the structural corrosion is addressed in EC2 (2005).

It is noted that the impact of climate change on man-made systems has a large component of uncertainty, given that the future climatic scenarios mainly depend on the evolution of the global sociopolitical context. Therefore, including climate change adaptation measures in the design phase of buildings and infrastructure is challenging because underestimating its impact would result in significant societal consequences, whereas overestimating it would imply an unnecessarily waste of resources. A resilience-based approach is proposed to address the issue, where the adaptation measures included in the design phase should tackle part of the impact of climate change, and during the service phase, upgrading mechanisms should be implemented to guarantee the system is prepared for the non-accounted impact of climate change if the evolution of climate change requires it

Therefore, this work aims to provide an overview of the problem of corrosion induced by climate change to better understand the need for a resilience-based approach to address the standardisation process. The general framework proposed for the adaptation to climate change by the new generation of structural Eurocodes is outlined and the associated challenges are further discussed.

The remaining document is organized as follows; Section 2 provides an overview of the

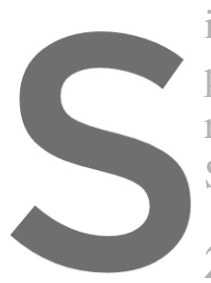
impact of climate cha

presents the corrosion resilient-based approact Section 5 some conclus
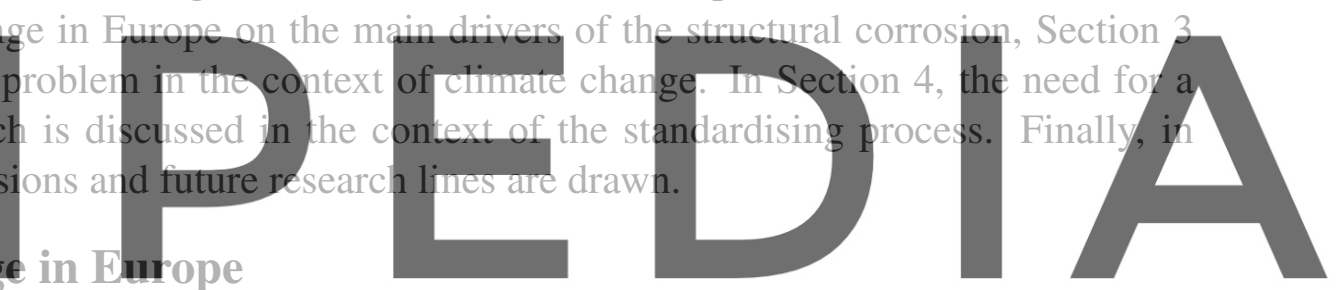

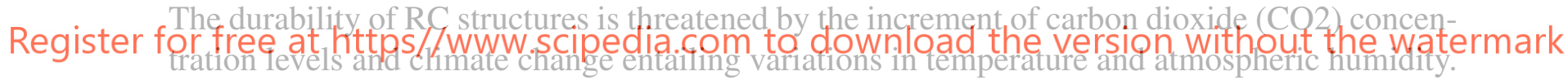

These three parameters are the principal environmental drivers of corrosion.

To study the expected variations of air temperature and relative humidity $(\mathrm{RH})$, the Representative Concentration Pathways (RCPs) proposed by IPCC (2014) are used. They are the so-called RCP2.6, RCP4.5, RCP6.0, and RCP8.5 scenarios, with RCP2.6 being closer to the scenario aimed by the Paris Agreement and RCP8.5 relating to the business-as-usual scenario. There exists a consensus amongst climatologists that RCP2.6 is naively optimistic, thus in practicality, the RCP4.5 is very often proposed as the potential optimistic scenario.

The definition of each scenario allows climatologists to determine the general atmosphere patterns and oceanic circulation dynamics at the global scale. Then, through complex processes of downscaling, climate changes at a regional level can be estimated with a significant level of confidence for each scenario providing a horizontal resolution of up to $0.11^{\circ}$ (about $12 \mathrm{~km}$ ).

In Europe, it is clear that climate change will increase air temperature. The extent of the variation will depend on the geographical location, period of the year, and scenario considered; for instance, North Europe, which is the most affected area when analysing air temperature, 
Table 1: Variation of air temperature due to climate change in Europe. The level of agreement among climatic models is given in brackets: L-low, M-medium, H-high, V-very high.

\begin{tabular}{lcccc}
\hline Area & Winter & Summer & Autumn & Spring \\
\hline & $(\mathrm{DJF})$ & \multicolumn{5}{c}{$(\mathrm{JJA})$} & $(\mathrm{SON})$ & $(\mathrm{MAM})$ \\
\hline \multirow{3}{*}{ North Europe } & \multicolumn{5}{c}{ RPC4.5 } \\
Central Europe & $2^{o}-8^{o}(\mathrm{M})$ & $0^{o}-6^{o}(\mathrm{H})$ & $2^{o}-6^{o}(\mathrm{H})$ & $2^{o}-6^{o}(\mathrm{M})$ \\
Mediterranean area & $0^{o}-4^{o}(\mathrm{H})$ & $0^{o}-4^{o}(\mathrm{H})$ & $0^{o}-4^{o}(\mathrm{M})$ & $0^{o}-2^{o}(\mathrm{~V})$ \\
East Europe & $0^{o}(\mathrm{H})$ & $2^{o}-4^{o}(\mathrm{H})$ & $0^{o}-4^{o}(\mathrm{H})$ & $0^{o}-4^{o}(\mathrm{H})$ \\
\hline \multirow{5}{*}{ North Europe } & $2^{o}-6^{o}(\mathrm{H})$ & $0^{o}-4^{o}(\mathrm{M})$ & $0^{o}-4^{o}(\mathrm{H})$ & $0^{o}-4^{o}(\mathrm{H})$ \\
\cline { 2 - 5 } Central Europe & $4^{o}-10^{o}(\mathrm{M})$ & $2^{o}-6^{o}(\mathrm{H})$ & $2^{o}-6^{o}(\mathrm{H})$ & $2^{o}-8^{o}(\mathrm{H})$ \\
Mediterranean area & $2^{o}-4^{o}(\mathrm{~V})$ & $2^{o}-4^{o}(\mathrm{~V})$ & $2^{o}-6^{o}(\mathrm{H})$ & $2^{o}-4^{o}(\mathrm{H})$ \\
East Europe & $2^{o}-4^{o}(\mathrm{~V})$ & $2^{o}-6^{o}(\mathrm{M})$ & $2^{o}-6^{o}(\mathrm{H})$ & $2^{o}-4^{o}(\mathrm{~V})$ \\
& $2^{o}-6^{o}(\mathrm{M})$ & $2^{o}-6^{o}(\mathrm{H})$ & $2^{o}-6^{o}(\mathrm{H})$ & $2^{o}-6^{o}(\mathrm{H})$ \\
\hline
\end{tabular}

presents increments ranging between $4^{\circ}-10^{\circ}$ during the winter months (December to February) under the RCP8.5, whereas the RCP4.5 provides increments of $2^{\circ}-8^{\circ}$ during winter. The range of values is given by the use of different climatic models. Table 1 summarises the variation of air temperature due to climate change in Europe.

The impact of climate change on the RH is smaller. Regardless of the climatic scenario considered, only during the summer months (JJA), Europe might experiment reductions of up

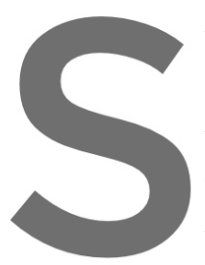
to $10 \%$ of the RH.

It is noted that an $i$ the uncertainty regardin which depends on fact this type of uncertainty
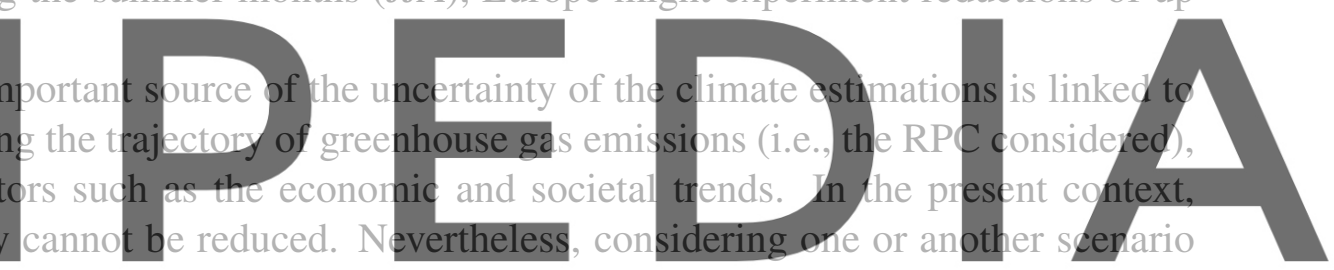

might differ in billion of euros in the long term. This should be kept in mind when updating the

Register foruffectaleqdęps//www.scipedia.com to download the version without the watermark

\section{Impact of Climate Change-Induced Corrosion}

\subsection{Corrosion Mechanisms}

$\mathrm{RC}$ corrosion refers to the phenomenon of the corrosion of the steel reinforcement of concrete caused by the infiltration into the concrete members of carbon dioxide, which reduces concrete $\mathrm{pH}$ till values below the steel passivisation threshold, and chloride, which reduces concrete alkalinity. Both processes are affected by temperature and $\mathrm{RH}$.

When studying the RC corrosion, the stage characterised by the ingress process of the $\mathrm{CO} 2$ and chloride ion into concrete is known as the initiation stage. In this stage, it is of interest to determine the chloride concentration at the rebar level and also when the carbonation reaches this level, that is, the carbonation depth.

Several studies have analysed the impact of climate change in the carbonation ingress process when assuming different $\mathrm{CO} 2$ levels, $\mathrm{RH}$, and temperature conditions. As shown in Table 2, the estimated increments of the carbonation depths are up to $45 \%$ by 2100 under the RCP 8.5 
Table 2: Effect of climate change on the carbonation and chlorine ion ingress processes in RC structures.

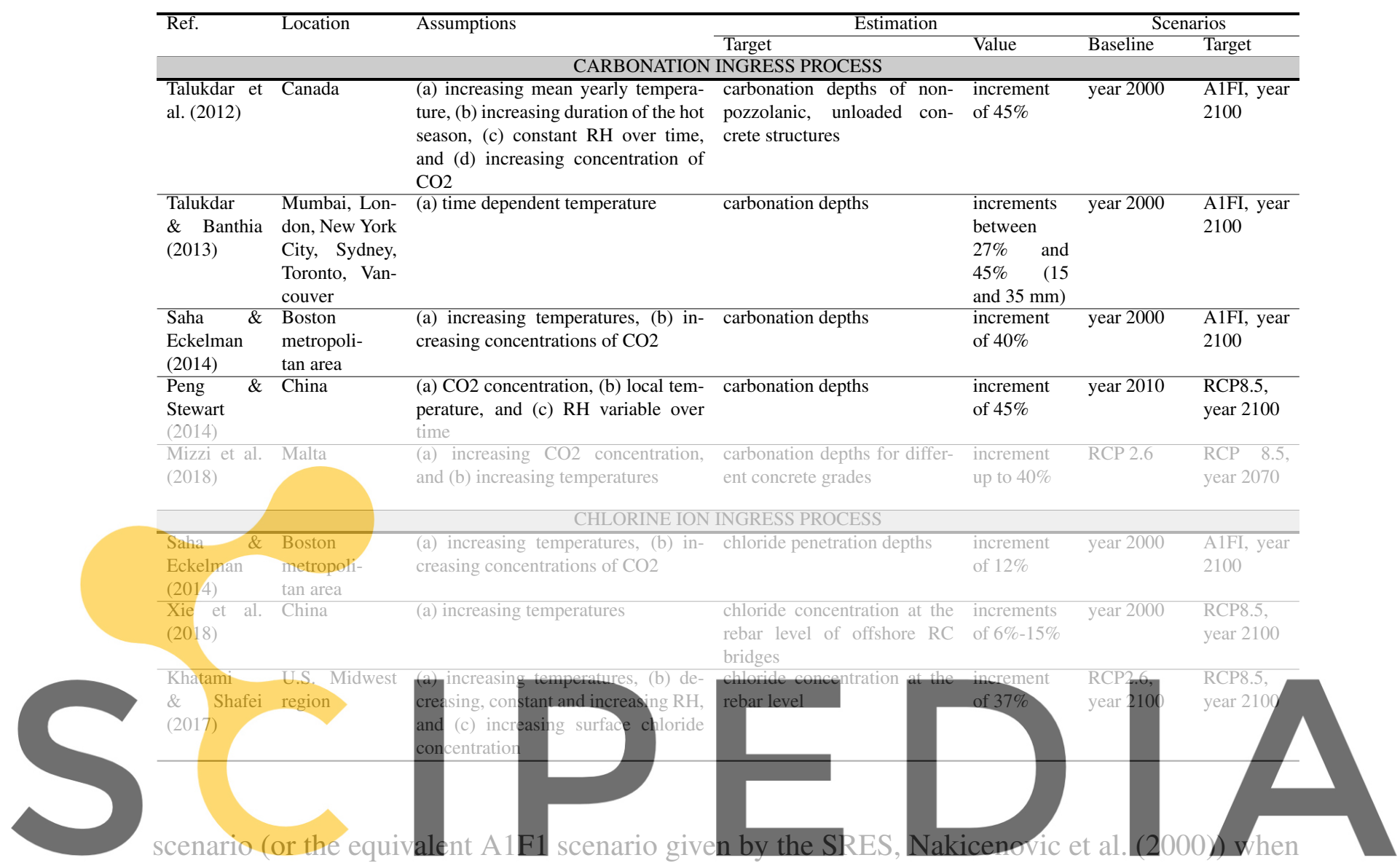

comparing with the year 2000. It is noted that the results are very sensitive to local exposure

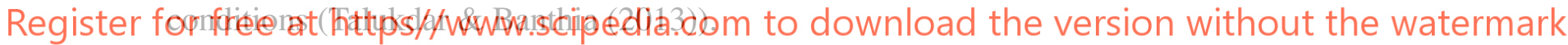

In the case of the impact of climate change to the chlorine ion ingress process, the studies

report more modest values, though still relevant, for the increment of chloride concentration at the rebar level when comparing the 2000 -year values to the RCP8.5 by the end of century, reaching values around $15 \%$ (see Table 2). Khatami \& Shafei (2017) report larger values, as they introduce in their model an increment of use of de-icing salts as a consequence of the observed consumption in the USA, which increased $200 \%$ in the last 25 years.

The second stage of the corrosion is known as propagation stage, which occurs once that the corrosion of the reinforcing steel is initiated, and finishes with the loss of steel area, cover cracking and spalling, and loss of the bond between steel and concrete. Whereas the initiation stage dominates the service life of the structure, the propagation stage lasts only a few years. For instance, Xie et al. (2018) report that the corrosion propagation stage of the offshore RC structures in China designed according to MOHURD (2008) would last less than one year considering future climatic conditions. 


\subsection{Impact on Reliability and Service Life}

As a consequence of climate change, both the reliability and service life of RC structures are highly impacted. The extent of this impact depends on the geographical location, the structural codes used for design, and the climatic scenario considered. Table 3 summarises some of the findings regarding the impact of climate change on the reliability and service life of RC structures by the end of this century if the most pessimistic scenario, i.e., the business-as-usual scenario, is considered. Even when the compound effect of the carbonation- and chlorinationinduced corrosion has not been studied yet, the enormous impact that climate change has on structural durability cannot be ignored.

Table 3: Impact of climate change on reliability and service life of RC structures.

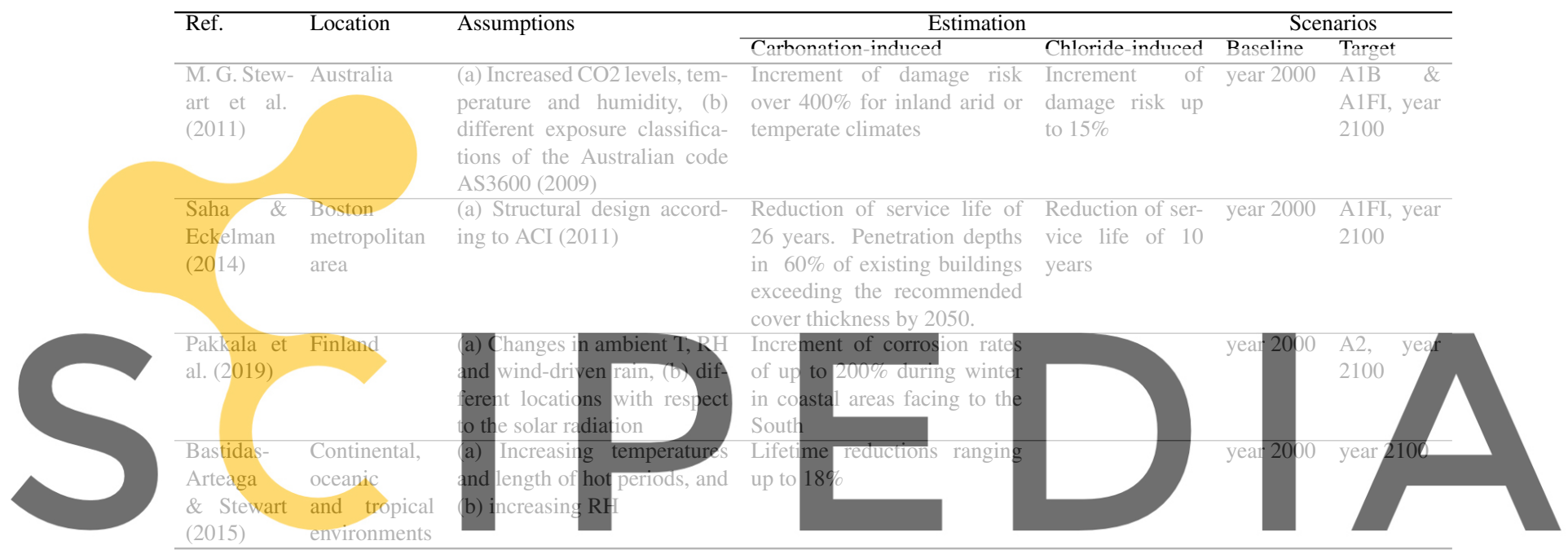

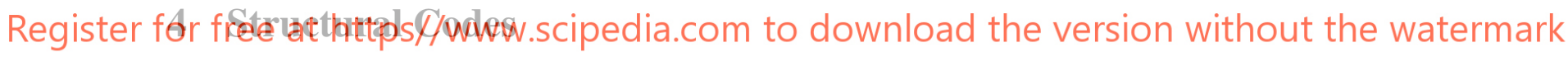

\subsection{Adaptation Measures}

The limit state of RC corrosion should include two phenomena, the cracking of concrete due to the sub-products generated by the corrosion and the reduction of effective steel cross-section, both occurring during the propagation stage. While the first is more related to aesthetic issues and the latter is linked to the structural integrity, the functionality of the structural member will determine which of the two phenomena is more critical. Given that the integrity of a structural member affected by corrosion will depend on the applied loads and structural configuration, its study becomes very case-specific and difficult to be generalised. Thus, when defining standardised strategies to adapt to climate change, it is better to focus on the initiation stage, which implies a larger portion of the structural service life and is less dependent on the applied loads and structural configuration.

Structural codes worldwide share a similar rationale when addressing the problem of corrosion; they focus on structural durability. Figure 1 generalises this approach. There exist two decision variables in regard to the structural design affecting the $\mathrm{RC}$ corrosion, that is, materials 


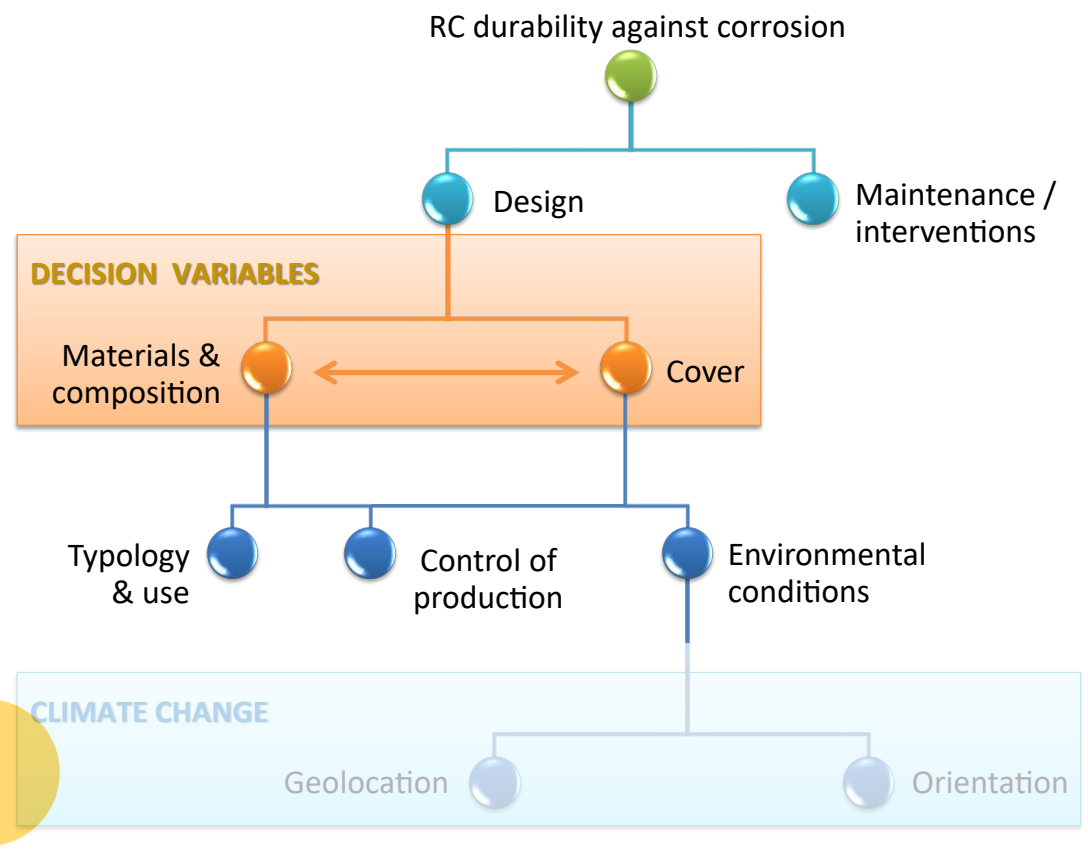

Figure 1: Rationale of structural codes to guarantee the structural durability against RC corrosion.

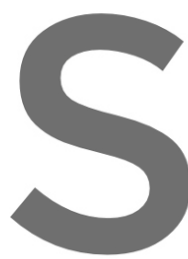

and composition and cor concrete allows for a reti typology and use, the c

ered. Initially, the new here.
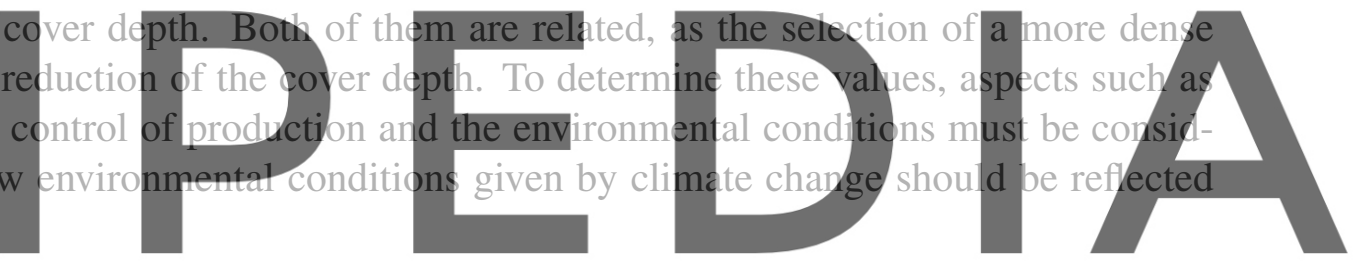

Regarding the materials and composition, two possible solutions can be taken, either improv-

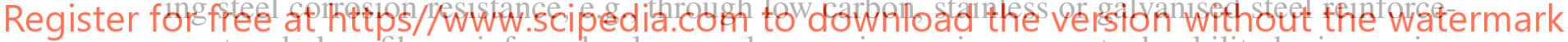 ment and glass-fiber reinforced polymer rebars, or improving concrete durability by increasing} the concrete grade, the water-cement ratio or applying concrete coatings. In addition, other concrete mixes, such as blended and alkali-activated (AA) cement, can be used that present higher density matrices than the standard and commonly used Portland cement (PC). The structure of their matrices reduces the permeability of the harmful substances. In addition, the AA slag cement, where the slag substitutes part of the PC, presents higher resistance to corrosion and its production generates lower greenhouse gas emissions than PC. Despite the slag cement has been used in the USA for more than 100 years, the feasibility of replacing PC blinders should be further investigated.

Nevertheless, it is estimated that the measures required to adapt to climate change, such as increasing design cover by up to $8 \mathrm{~mm}$ or increasing concrete compressive strength by one grade would imply an increment of 1-3\% of the construction costs (M. Stewart et al. (2012)). Other authors (Bastidas-Arteaga \& Stewart (2016); M. Stewart \& Bastidas-Arteaga (2019)) have addressed the cost-benefit analysis of increasing the cover thickness to 5 or $10 \mathrm{~mm}$ in 
different structural components under different climate scenarios. They conclude that in many cases, this measure is not cost-effective. The factors affecting the efficiency of this type of adaptation measures do not depend only on the structural typology and member size, but also on the climatic conditions and the future discount rate.

Keeping in mind the impact that Eurocodes has in the economy of the State Members, standardising adaptation measures in a context of high uncertainty, where if the impact of climate change is underestimated by considering very optimistic scenarios, it would result in a significant reduction of the service life of new infrastructure and buildings, or if it is overestimated, in unnecessarily expensive investment. Apparently, there is not a fair solution.

\subsection{Resilience-Based Approach}

In the last years, there has been a clear shift from the traditional risk-oriented approaches to a perspective based on resilience. The need for a resilience-based approach has been made evident in those cases where either, the lack of knowledge about the potential hazards or their level of uncertainty was so large that the existing risk was underestimated. For that reason, the problem of climate change along with its associated uncertainty is pushing decision-makers to implement measures that boost resilience (Nogal \& O'Connor (2018a); Val et al. (2019)). In this point it is important to note that risk- and resilience-based approaches are not incompatible; on the contrary, the latter complements the first one by adding a temporal dimension to the

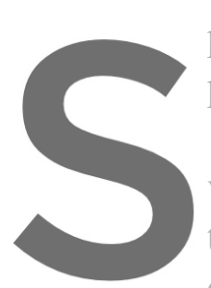
problem (Nogal \& O'Connor $(2018 \mathrm{~b})$ ) and proposing a number of strategies to increase the
preparedness level of the system and so allow for an adaptive response over time.
Therefore, to apprdach the issue of climate-change induced corrosion from a resilence-based
view, the adaptation measures should be twofold. On the one hand, the measures addressing
the impact of climate change that is certain should be implemented in the design stage, throdgh
cost-effective measures. More sustainable concrete mixtures might play an important role in this stage. On the other hand, the uncertain impact of climate change should be covered in a post-

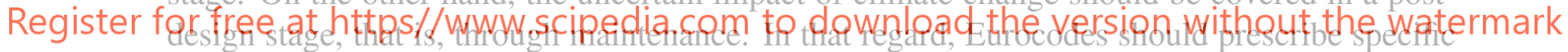

maintenance in relation to climate change. It is clear that there is room for improvement in terms of maintenance in the present standards, as they merely define maintenance, mentioning that the structure will be adequately maintained.

Such an approach implies a number of challenges. The values of $\mathrm{CO} 2$ concentration levels, temperature and atmospheric humidity associated with low values of uncertainty should be determined based on the climate models for the end of the structural design life. Let's call them certain thresholds (CTs) of the corrosion drivers.

The maintenance strategies, which should be studied during the design phase, should state the frequency of the maintenance activities in which the updated information regarding climate change will be used to determine the short- and medium-term actions. Also at the design phase, a number of potential actions should be included considering the future climatic scenarios along with their economic cost. Finally, an economic program to face the potential structural upgrades should be included to guarantee the economic viability of the project. It is noted that some of the required maintenance interventions are usually less cost-effective than the measures adopted 


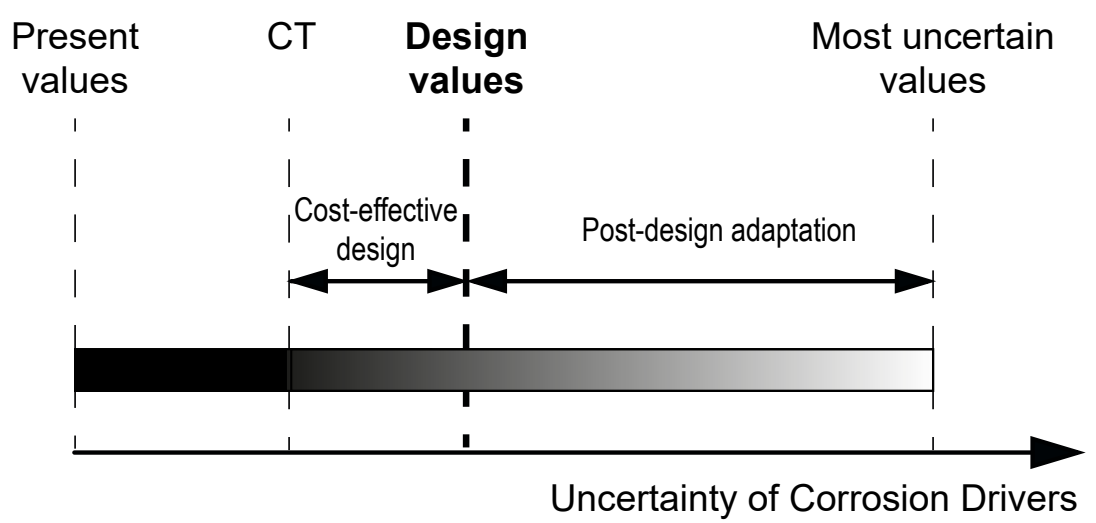

Figure 2: Representation of the design value of the corrosion drivers based on the cost-effectiveness of the adaptation measures and the uncertainty regarding the future value of the corrosion driver.

in the initial design. Therefore, the most cost-effective design should not cover only the CTs of the corrosion drivers, but more uncertain values in order to minimise the life cycle cost (see Figure 2). In other words, the new generation of Eurocodes should provide a design value of the corrosion drivers that will be established based on both, the uncertainty regarding the future value of the corrosion driver and the cost-effectiveness of the adaptation measures.
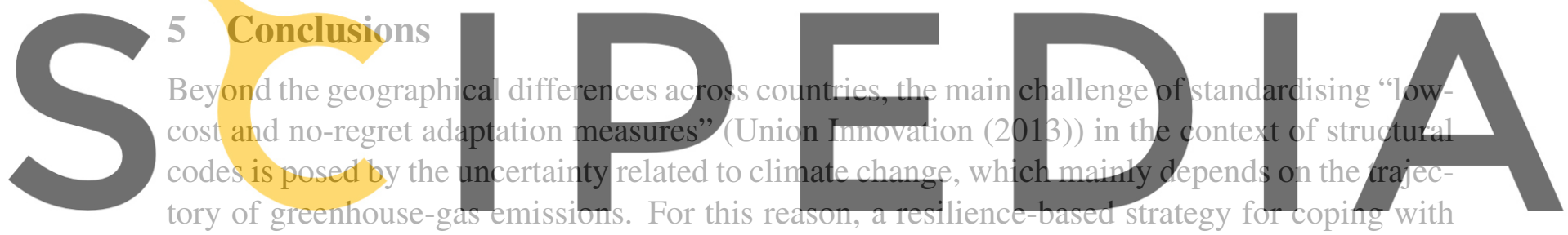

climate change-induced corrosion has been suggested in this paper. Such an approach aims to

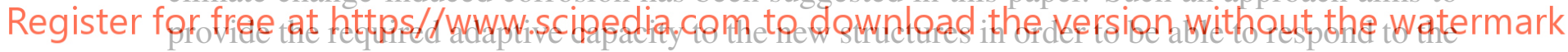

uncertain future minimising the investment under unlikely scenarios. In that way, the strategy

can be summarised as a climate change-adapted design plus an in-design maintenance plan. It is highlighted the need for an improvement of the structural Eurocodes in the area of maintenance.

Although the impact of climate change on temperature and atmospheric humidity is of interest in the context of $\mathrm{RC}$ corrosion, the $\mathrm{CO} 2$ levels should be also considered. The maintenance activities, which will be defined according to the on-going weather-related variables and $\mathrm{CO} 2$ emissions will further motivate countries to reduce their $\mathrm{CO} 2$ emissions in order to minimise their medium and long-term investments. Note that whereas climate change is a global issue, $\mathrm{CO} 2$ concentrations have an important local component, and thus, the proposed strategy will penalize more heavily those areas producing a larger amount of $\mathrm{CO} 2$.

This paper has shown a general framework for the adaptation of structural codes to climate change-induced actions under a resilience-based approach. Specific actions, such as mapping the future climate-related drivers of corrosion, the determination of the design values of these drivers, and the prescription of maintenance activities, should be further investigated. 


\section{ORCID}

Maria Nogal: http://orcid.org/ 0000-0001-5405-0626

\section{References}

ACI. (2011). Building code requirements for structural concrete and commentary..

AS3600. (2009). AS3600 (2009), concrete structures. Standards Australia, Sydney.

Bastidas-Arteaga, E., \& Stewart, M. (2015). Damage risks and economic assessment of climate adaptation strategies for design of new concrete structures subject to chloride-induced corrosion. Structural Safety, 52, 40-53.

Bastidas-Arteaga, E., \& Stewart, M. (2016). Economic assessment of climate adaptation strategies for existing reinforced concrete structures subjected to chloride-induced corrosion. Structure and Infrastructure Engineering, $12(4), 432-449$.

EC2. (2005). En 1992 eurocode 2: Design of concrete structures. Brussels: CEN.

IPCC. (2014). Climate change 2014: svnthesis report. IPCC Geneva, Switzerland.

Khatami, D., \& Shafei, B. (2017). Climate change impact on management of deteriorating bridges: A case study of US Midwest Region (Tech. Rep.).

Mizzi, B., Wang, Y., \& Borg, R. P. (2018). Effects of climate change on structures; analysis of carbonationinduced corrosion in reinforced concrete structures in Malta. In Iop conference series: Materials science and engineering (Vol. 442, p. 012023).

MOHURD. (2008). Code for durability design of concrete structures..

Nakicenovic, N., Alcamo, J., Grubler, A., Riahi, K., Roehrl, R., Rogner, H.-H., \& Victor, N. (2000). Special report on emissions scenarios (SRES), a special report of Working Group iii of the intergovernmental panel on climate

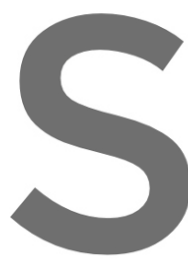
change. Cambridge Unive

Nogal, M., \& O'Connor, resilience for complex

Nogal, M., \& O'Connor, Sustainable and Resilient Infrastructure, 199

Pakkala, T. A., Köliö, A., Lahdensivu, J., \& Pentti,
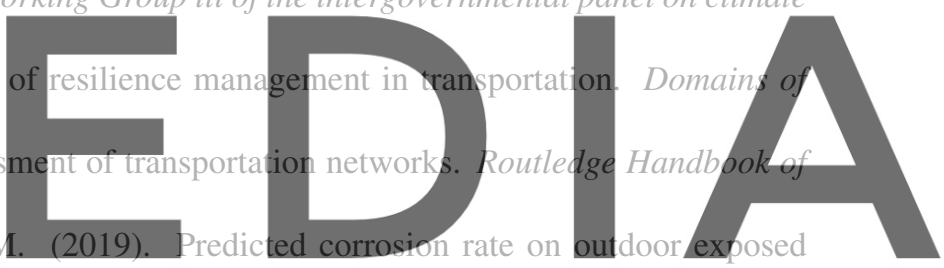
concrete structures. International Journal of Building Pathology and Adaptation.

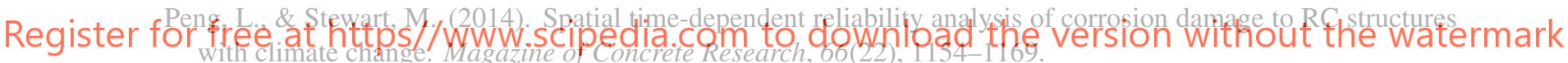

Saha, M., \& Eckelman, M. J. (2014). Urban scale mapping of concrete degradation from projected climate change. Urban Climaie, 9, 101-114.

Stewart, M., \& Bastidas-Arteaga, E. (2019). Corrosion of concrete and steel structures in a changing climate. In Climate adaptation engineering (pp. 99-125). Elsevier.

Stewart, M., Wang, X., \& Nguyen, M. (2012). Climate change adaptation for corrosion control of concrete infrastructure. Structural Safety, 35, 29-39.

Stewart, M. G., Wang, X., \& Nguyen, M. N. (2011). Climate change impact and risks of concrete infrastructure deterioration. Engineering Structures, 33(4), 1326-1337.

Talukdar, S., \& Banthia, N. (2013). Carbonation in concrete infrastructure in the context of global climate change: development of a service lifespan model. Construction and Building Materials, 40, 775-782.

Talukdar, S., Banthia, N., Grace, J., \& Cohen, S. (2012). Carbonation in concrete infrastructure in the context of global climate change: Part 2- Canadian urban simulations. Cement and Concrete Composites, 34(8), 931-935.

Union Innovation. (2013). Communication from the Commission to the European Parliament, the Council, the European Economic and Social Committee and the Committee of the Regions. A new skills agenda for Europe. Brussels.

Val, D. V., Yurchenko, D., Nogal, M., \& O'Connor, A. (2019). Climate change-related risks and adaptation of interdependent infrastructure systems. In Climate adaptation engineering (pp. 207-242). Elsevier. 
Xie, H., Wang, Y., Gong, J., Liu, M., \& Yang, X. (2018). Effect of global warming on chloride ion erosion risks for offshore RC bridges in China. KSCE Journal of Civil Engineering, 1-7.
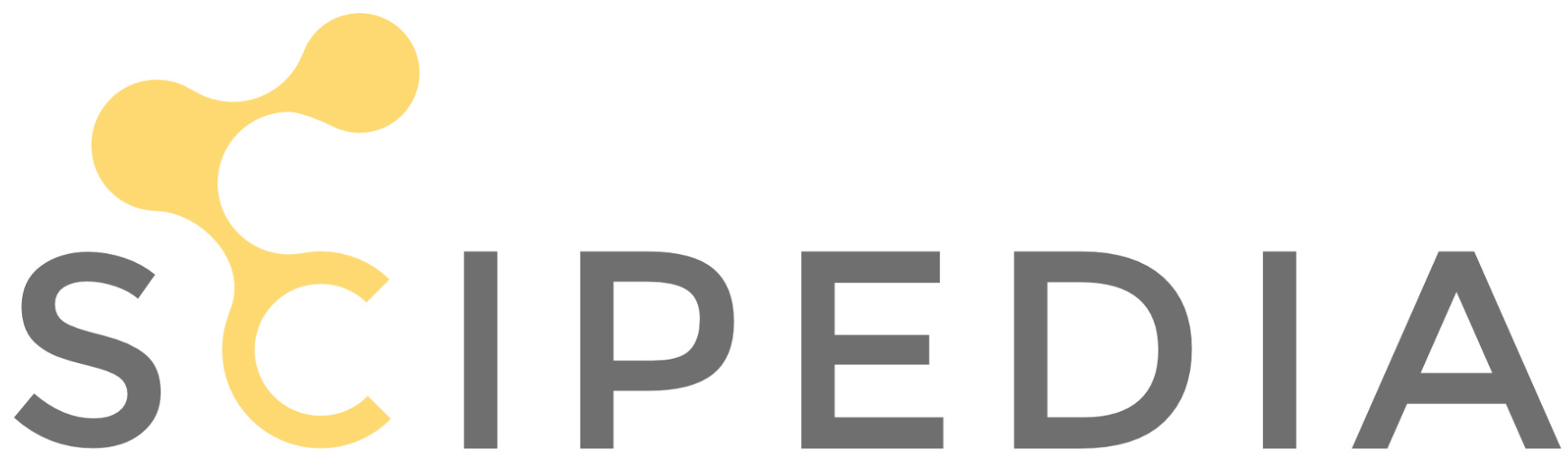

Register for free at https//www.scipedia.com to download the version without the watermark 